\title{
MALLOCHIANAMYIA, NOME NOVO PARA GAYOMYIA MALLOCH (DIPTERA, CHAMAEMYIIDAE) ${ }^{1}$
}

\author{
Luiz Gonzaga dos Santos-Neto ${ }^{2}$
}

\begin{abstract}
Mallochianamyia, NeW name for Gayomyla Malloch (Diptera, ChamaemyiddaE). A brief bibliography of Gayomyia Banks, 1913 (Neuroptera, Hemerobiidae) and of Gayomyia Malloch, 1933 is presented.

KEY WORDS. Gayomyia, homonymy, Mallochianamyia, new name
\end{abstract}

São apresentados dois homônimos: Gayomyia Banks, 1913 (Neuroptera, Hemerobiidae) e Gayomyia Malloch, 1933 (Diptera, Chamaemyiidae). A etimologia deste nome não é explicada pelos autores, mas provavelmente eles homenagearam a mesma pessoa, o naturalista chileno Claudio Gay (1800-1873).

BANKS (1913: 217) descreveu o gênero Gayomia para Megalomus falcatus Blanchard, 1851. Porém, uma segunda grafia desse nome apareceu na página anterior como Gayomyia em uma chave dicotômica.

Handschin (1955: 6), Penny (1977: 30) e Oswald \& Penny (1991: 26), utilizaram a grafia Gayomyia, atuando os autores do último artigo como o "primeiro revisor" (ICNZ 1985, artigo 24b). Este gênero foi recentemente revisado por OSWALD (1993).

MALLOCH (1933: 346) descreveu o gênero e a espécie Gayomyia nigrohalterata (Diptera), do Chile, em família duvidosa. PRADo (1984: 1) incluiu o gênero em Pallopteridae. MCALPINE (1987: 966) e ColLESS \& MCALPINE (1991: 769) o mencionaram, com dúvida, em Chamaemyiidae, sendo que os últimos artigos citam a ocorrência deste gênero na Austrália.

Assim um nome novo é proposto: Mallochianamyia, nom.nov. para Gayomyia Malloch, 1933 (non Banks, 1913), em homenagem a John R. Malloch.

\section{REFERÊNCIAS BIBLIOGRÁFICAS}

BAnKs, N. 1913. Synopses and descriptions of exotic Neuroptera. Trans. Am. Ent. Soc. 39 : 201-242.

Colless, D.H. \& D.K. MCAlPINE. 1991. Diptera (Flies), p.717-786. In: CSIRO (Ed.). The Insects of Australia. Melbourne, Melbourne University Press, $2^{\text {nd }}$ edition, vol. 2, 1137p.

Handschin, E. 1955. Los insectos de las Islas Juan Fernandez. 15. Neuroptera. Rev. Chil. Entom. 4: 3-20.

1) Contribuição número 903 do Departamento de Zoologia, Universidade Federal do Paraná.

2) Departamento de Zoologia, Universidade Federal do Paraná, Caixa Postal 19020, 81531-990 Curitiba, Paraná, Brasil. 
ICZN. 1985. International Code of Zoological Nomenclature. London, $3^{\text {rd }}$ ed., $338 \mathrm{p}$.

MCAlPINE, J.F. 1987. Chamaemyiidae, p.965-971. In: J.F. MCAlpine (Ed.)

Manual of Nearctic Diptera. Ottawa, Biosystematics Research Centre, Monograph no. 28, vol. 2, 1332p.

Malloch, J.R. 1933. Diptera of Patagonia and South Chile. 6. Acalyptrata. London, British Museum (Natural History), p.177-391.

OSWALD, J.D. 1993. Revision and cladistic analysis of the world genera of the family Hemerobiidae (Insecta: Neuroptera). Jour. N.Y. Entomol. Soc. 101 (2): 143-299.

Oswald, J.D. \& N.D. PENNY. 1991. Genus-group names of the Neuroptera, Megaloptera and Raphidioptera of the world. Occ. Pap Calif. Acad. Scienc. 147: 1-94.

PENNY, N.D. 1977. Lista de Megaloptera, Neuroptera e Raphidioptera do México, América Central, ilhas Caraíbas e América do Sul. Acta amazonica 7 (supl.): $1-61$.

PRADO, A.P. DO. 1984. 70. Family Pallopteridae. In: A Catalogue of the Diptera of the Americas South of the United States. São Paulo, Museu de Zoologia, Universidade de São Paulo, 2p. 